\title{
Dependence of Electrical Properties on Frequency and Temperature for Novel Cyclopentenone Derivatives
}

\author{
Amal M. Abdel-Karim ${ }^{1}$, A. H. Salama ${ }^{1 *}$, Fatma A. El-Samahy ${ }^{2}$ \\ ${ }^{1}$ National Research Centre, Department of Physical Chemistry, 33 El Bohouth St. ( El-Tahrir St. former) - Dokki, Giza, Egypt \\ ${ }^{2}$ National Research Centre, Department of Green Chemistry, 33 El Bohouth St. (El-Tahrir St. former) - Dokki, Giza, Egypt \\ E- mail: alia2005salama@yahoo.com
}

\begin{abstract}
Electrical properties of some new cyclopentenone derivatives have been studied. The structures of prepared samples were characterized by (UV), (XRD) and (SEM). The dependence of electrical properties such as $\sigma_{\mathrm{dc}}, \sigma_{\mathrm{ac}}, \varepsilon^{\prime}$ and $\varepsilon^{\prime \prime}$ on frequency and temperature were studied at frequency range from $50 \mathrm{~Hz}$ to $5 \mathrm{MHz}$ and the temperature range from $25^{\circ} \mathrm{C}$ to $140^{\circ} \mathrm{C}$. It was found that, $\varepsilon^{\prime}$ decreased with increasing frequency while it increases with increasing temperatures within the used ranges. Moreover, dielectric constant is structural dependent which is obvious from the variation of dielectric constant for each sample. Ac-electrical conductivity increased with increasing frequency which was attributed to the polarization of the charge carriers. The temperature dependence of dc-electrical conductivity show typical Arrhenius relation for the three prepared samples. The activation energy calculated from Arrhenius equation and the results are discussed in detailed.
\end{abstract}

Key words: organic compounds, preparation, cyclopentenone derivatives, dc- and ac-electrical conductivity, dielectric properties

\section{Introduction}

Now days the scientists and engineers devoted their attention to study the different types of organic compounds especially $\pi$-conjugated one to shed light on its semiconducting properties. Organic semiconductors (OS) vary from inorganic one in several ways, for example electronic, optical, structural and chemical properties. OS gained scholars attention because of their potential to replace inorganic solar cells due to their lightweight, low-cost and easy fabrication and its importance in many applications as organic light-emitting diodes (OLED), electrochemical transistors and recently in bio-sensor applications ${ }^{[1,2]}$. Literature review show that there is only a few studies on the dielectric behavior which gives information about the distribution of electric field within the solid ${ }^{[3]}$ and electric conductivity on organic compounds. Low and high dielectric constant are essential in electronic industrial sectors. Low dielectric constant is required basically as insulators where a material with high dielectric constant is highly desired to satisfy the requirements in high energy density capacitors. It is also used for applications in low fire multilayer devices and tunable devices from high frequency to microwave range. The properties of dielectric materials with high dielectric constant make them a new candidates for voltage-tunable devices such as tunable filter, tunable antenna and phase shifter, etc.

Cyclopentenones are key building blocks for organic synthesis and possess many biological properties ${ }^{[4]}$ The cyclopentenone ring is present in a broad collection of interesting drug targets, natural ${ }^{[5]}$ and commercial products ${ }^{[6]}$. Also Pyrazoles and their derivatives are well established in the literature as significant biologically active heterocyclic compounds $^{[7]}$. The pyrazole nucleus (aromatic, two nitrogen atoms in five member ring) exists in a variety of biologically and pharmaceutical attractive compounds. ${ }^{[8]}$

Consequently, the synthesis of this type of compounds and a new derivative has attracted considerable attention. This feature is not only important from a fundamental scientific view point but also for its possible applications wherever bipolar organic compounds are encouraging candidates to develop further development of the field associated with organic electronics ${ }^{[9]}$.

Organic electronics is important in many fields of research. The quick progress attention provided to $\pi$-conjugated materials by both academics and industry semiconducting conjugated polymers have been of superb important in organic 
electronics applications ${ }^{[10]}$ photovoltaic cells ${ }^{[11]}$ photocatalyst polymers ${ }^{[12]}$ and organic batteries ${ }^{[13]}$.

In the present work, the electrical properties as dc-and ac-electrical conductivity, dielectric constant and dielectric loss of three different prepared samples 1, 2 and 3 are studied at various frequency and temperature ranges after characterization by UV-Vis spectra, XRD and SEM. Band gap energy values of these organic compounds are calculated from UVVis. spectra. Grain size are calculated from XRD and compared with that measured by SEM. The activation energy is calculated from the dependence of electrical conductivity on temperature by using Arrhenius equation. Thermal parameters as enthalpy and entropy changes had been calculated from the value of relaxation times estimated from the dielectric loss dependent on frequency curves.

\section{Experimental}

\subsection{Preparation of cyclopentenone derivatives 1, 2 and 3}

3, 4-Diphenyl-4-hydroxy-2-cyclopentenone (sample 1) was synthesized as previously described in the literature ${ }^{[14]}$. While the synthesis of 1,3,6,6a-tetraphenyl-1,6a-dihydrocyclopenta[c]pyrazol-4(3aH) one (sample 2) and 1-(3-Chlorophenyl)-3,6,6a-triphenyl-1,6a-dihydrocyclopenta[c]pyrazol-4(3aH)-one (sample 3) were synthesized using literature $^{[15]}$. To a mixture of sample $1(5 \mathrm{~m}$ mole $)$, hydrazones chlorides $(5 \mathrm{mmole})$ and triethylamine $(1.5 \mathrm{~mL})$ in dry benzene was heated under reflux. Triethylamine chlorohydrate was removed by filtration, the solvent was evaporated in vacuum, and the residue was chromatographer on silica gel to give sample 2 . The same procedure was repeated with preparation of 1-(3-chlorophenyl)-3, 6, 6a-triphenyl-1, 6a-dihydrocyclopenta[c]pyrazol-4(3aH)-one (sample 3). The structures elucidated by spectroscopic techniques as well as elemental analyses and molecular weights were determinate.

\subsection{Characterizations studies}

\subsubsection{UV-Vis spectroscopy}

UV-Vis spectroscopy was used to characterize the optical absorption properties of the prepared compounds. Absorption spectra were recorded in the wavelength range of 190 to $800 \mathrm{~nm}$ using a JASCO V360 UV-visible spectrometer. All spectra were recorded at room temperature. From the UV-Vis Spectra we can calculate the band gap energy (E, eV) by using the following equation ${ }^{[16]}$.

$$
\mathrm{E}=\mathrm{h}(\mathrm{c} / \lambda)
$$

Where, $\mathrm{h}$ is Plank' s constant $6.626 \times 10^{-34}$ Joules sec, $\mathrm{C}$ is Speed of light $3.0 \times 10^{8}$ meter $/ \mathrm{sec}, \lambda$ is the Cut off wavelength, 1 $\mathrm{eV}=1.6 \times 10^{-19}$ Joules.

\subsubsection{Ray diffraction}

$\mathrm{X}$-ray diffractograms provided with computer controlled X-ray diffractometer formally made by PHILIPS-MPD X PERT equipped with copper tube operating at $40 \mathrm{KV}$ and $30 \mathrm{~mA}$. From the well known Scherer formula equation (2), the average crystallite size, $\mathrm{L}$, is

$$
\mathrm{L}=(\mathrm{K} \lambda) /(\beta \operatorname{Cos} \theta)
$$

Where $\lambda$ is the $\mathrm{X}$-ray wavelength in the nanometer, $\mathrm{k}$ (constant equal to 0.9 ), and $\beta$ is the peak width at half maximum height. The value of $\beta$ in the $2 \theta$ axis of diffraction profile must be in radians. The $\theta$ is the Bragg angle and can be in radians since the $\cos \theta$ corresponds to the same number.

\subsubsection{Scanning electron microscope}

The scanning electron microscope (SEM) images were studied for the prepared samples using a JEDL model 1230.

\subsection{Electrical properties}

Dielectric properties of the respective compounds have been investigated on the form of pellets of about $10 \mathrm{~mm}$ in diameter which prepared by pressing powdered samples at $10 \mathrm{tons} / \mathrm{cm}^{2}$. The electrical properties were performed by using HIOKI 3532-50 LCR-Hi Tester. The capacitance data was employed to derive the corresponding real part of the dielectric constant as a function of frequency and temperature. The dielectric constant $\varepsilon^{\prime}$ is given by

$$
\varepsilon^{\prime}=(\mathrm{Cd}) /\left(\varepsilon_{0} \mathrm{~A}\right)
$$

Where $\mathrm{C}$ is measured capacitance, $\varepsilon_{0}$ is the permittivity of free space $\left(8.85 \times 10^{-12} \mathrm{~F} / \mathrm{m}\right)$ and $\mathrm{A}$ is the electrode area and $\mathrm{d}$ is 
the thickness.

The imaginary part of the dielectric function was estimated according to the relation

$$
\varepsilon^{\prime \prime}=\varepsilon^{\prime} \tan \delta
$$

The frequencies corresponding to the maximum energy loss $F_{m}$ are used to calculate the relaxation times from the relation

$$
\tau=1 /\left(2 \pi \mathrm{F}_{\mathrm{m}}\right)
$$

The activation enthalpies $\Delta \mathrm{H}$ and the entropy changes $\Delta \mathrm{S}$ of dielectric relaxation has been calculated from the slope and intercept of the plot of $\ln (\mathrm{T} \tau \mathrm{K}) / \mathrm{h}$ against $1 / \mathrm{T}$ of equation

$$
\operatorname{Ln}(\mathrm{T} \tau \mathrm{K}) / \mathrm{h}=\Delta \mathrm{H} / \mathrm{T}-\Delta \mathrm{S} / \mathrm{T}
$$

The frequency and temperature dependence of ac - conductivity $\left(\sigma_{\mathrm{ac}}\right)$ are determined from the measured values of the resistance for the sample under test. The ac - conductivity was calculated using the relation

$$
\sigma \mathrm{ac}=\mathrm{L} / \mathrm{R}_{\mathrm{p}} \mathrm{a}
$$

Where $R_{p}$ is the sample resistance, a is the cross sectional area of the sample and $L$ is the thickness of the sample. Then calculating frequency exponent $\mathrm{S}$ from power low relation

$$
\sigma_{\mathrm{ac}}=\mathrm{A} \omega^{\mathrm{S}}
$$

Where $\sigma_{\mathrm{ac}}$ is the ac-conductivity, $\omega$ is the angular frequency, $\mathrm{A}$ is constant, $\mathrm{S}$ is frequency exponent.

For dc-electrical conductivity studies, the dependence of conductivity on temperature were determined by plotting $\log \sigma$ versus 1000/T we found that it obeys Arrhenius relationship:

$$
\sigma=\sigma_{o} \exp \left(-\Delta \mathrm{E}_{\mathrm{a}} / \mathrm{KT}\right)
$$

Where, $\sigma_{\mathrm{o}}$ is the pre-exponential constant corresponding to $1 / \mathrm{T}=0, \mathrm{~T}$ is the absolute temperature and $\mathrm{K}$ is Boltzmann constant $\Delta \mathrm{E}$ is the activation energy which was calculated from the slope

$$
\Delta \mathrm{E}_{\mathrm{a}}=- \text { (slope) } \mathrm{k} \times 1000
$$

\section{Results and discussion}

The cyclopentenone derivatives (samples 1-3) were prepared by the interaction of 3, 4 -Diphenyl-4-hydroxy-2cyclopentenone (sample1) with hydrazonyl chlorides and triethylamine and characterized by FTIR spectroscopy, NMR and mass spectra according to our previously work ${ }^{[15]}$. Fig. (1) represents the structural formula of the prepared samples.

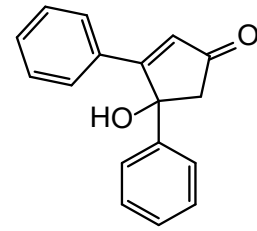

1

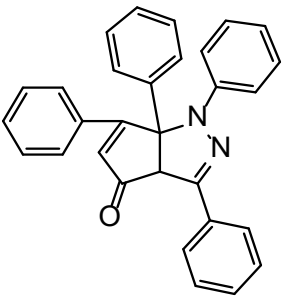

2

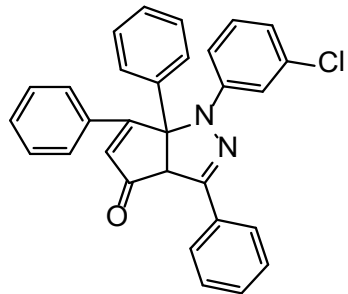

3

Figure 1. The structural formula of the prepared samples

\subsection{UV-visible spectra}

To study the optical properties of the samples 1, 2 and 3, the UV-visible spectra were recorded as shown in Fig (2). The spectra show good absorption properties with strong peak at $382 \mathrm{~nm}$ for sample 1 shifted to higher wavelength 
(bathochromic shift) at 399 and 413 for samples 2 and 3, respectively. This red shift can be attributed to transfer of electrons from $\pi$ or non-bonding orbital to unoccupied molecular orbital of greater potential energy resulting from increase the length of $\pi$-system through resonance. The band gap energy listed in table (1) is calculated using equation (1) ${ }^{[17]}$ by extrapolations of linear part of Fig. (2).

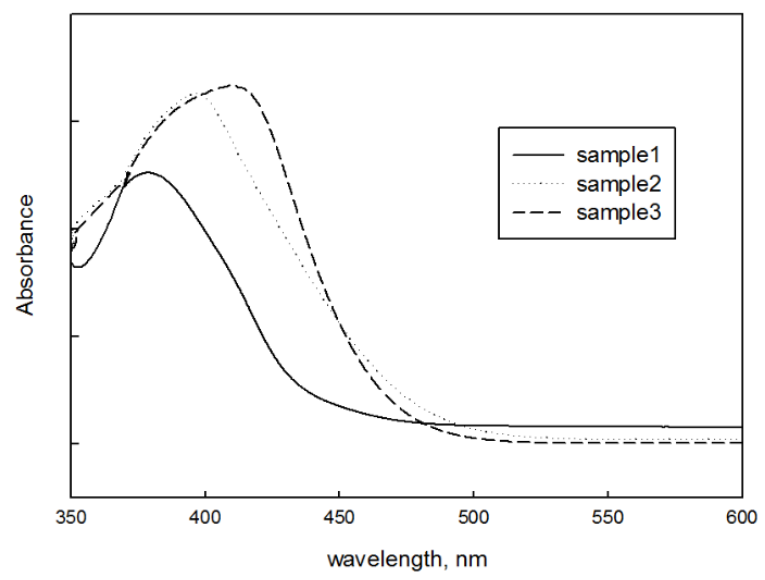

Figure 2. Uv-vis spectra of samples 1,2 and 3

Table 1. The values of energy gap calculated from UV spectra

\begin{tabular}{ccc}
\hline Compounds & Cut off $\lambda(\mathrm{nm})$ & Energy gap $(\mathrm{eV})$ \\
\hline Sample 1 & 433 & 2.86 \\
Sample 2 & 472 & 2.60 \\
Sample 3 & 465 & 2.67 \\
\hline
\end{tabular}

Substituent within the benzene ring causes bathochromic and hypsochromic shifts of various peaks. However, qualitative understanding of the effects of substituent on the characteristics of UV-vis spectrum can be considered by classifying the substituent into electron-withdrawing and electron-donating group. As the number of conjugated double bonds in sample $\mathbf{2}$ and $\mathbf{3}$ are increased, the gap between highest occupied molecular orbital (HOMO) and lowest unoccupied molecular orbital (LUMO) is lowered. So shifts the absorption maximum to longer wavelength .Regarding to the values of band gap energy recorded in table (1), it is obvious that the highest value was recorded for sample 1 . The lowest value was recorded for sample 2, where the presence of two nitrogen atoms and conjugated double bonds facilate the movement of electrons along the structure.

\subsection{X-ray diffraction}

The inner arrangement of atoms in the crystals of samples 1, 2 and 3 were investigated by XRD as shown in Fig. (3). It is obvious that the line width decreased in sample 2 and 3 indicated that the samples were homogeneous and are highly crystalline structure. By using Scherrer equation (2), the grain size of each sample was calculated ${ }^{[18]}$. We found the grain size of the prepared samples is $31.6 \mathrm{~nm}$ for sample $1,135 \mathrm{~nm}$ for sample 2 and $134 \mathrm{~nm}$ for sample 3 . 

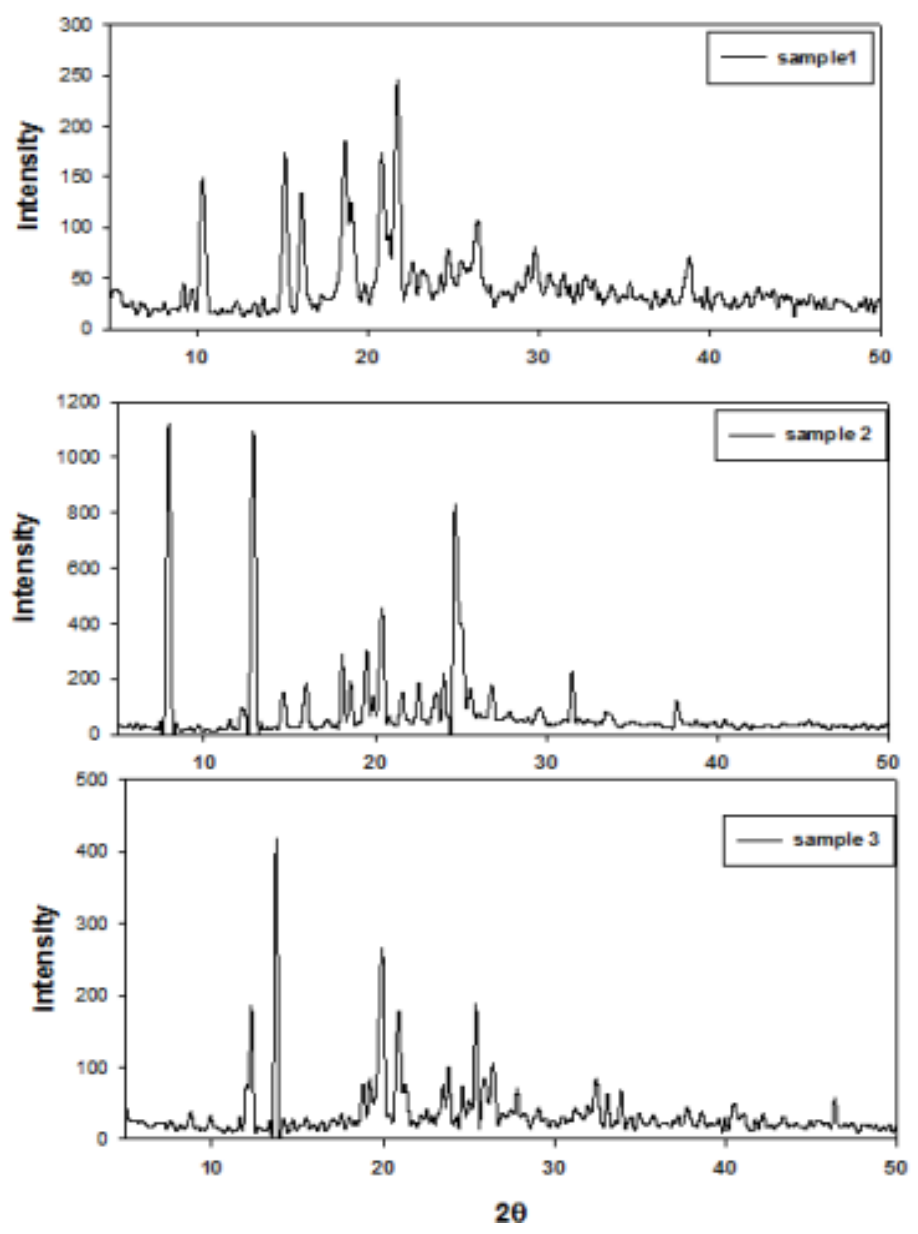

Figure 3. XRD pattern for samples 1, 2 and 3

\subsection{Scanning electron microscope}

Fig. (4) shows SEM image of the prepared samples. It is shown that the particles of samples 1 and 2 are plates like shape while sample 3 has a needle like structure. We found the grain size of each sample is in the same range as calculated from XRD spectra.

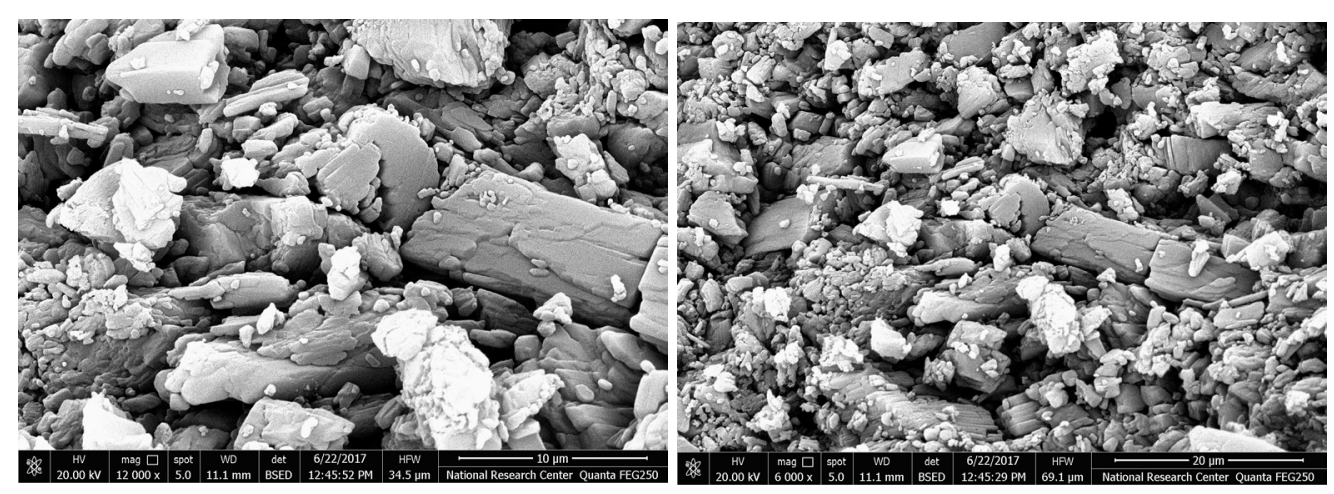

Sample 1 

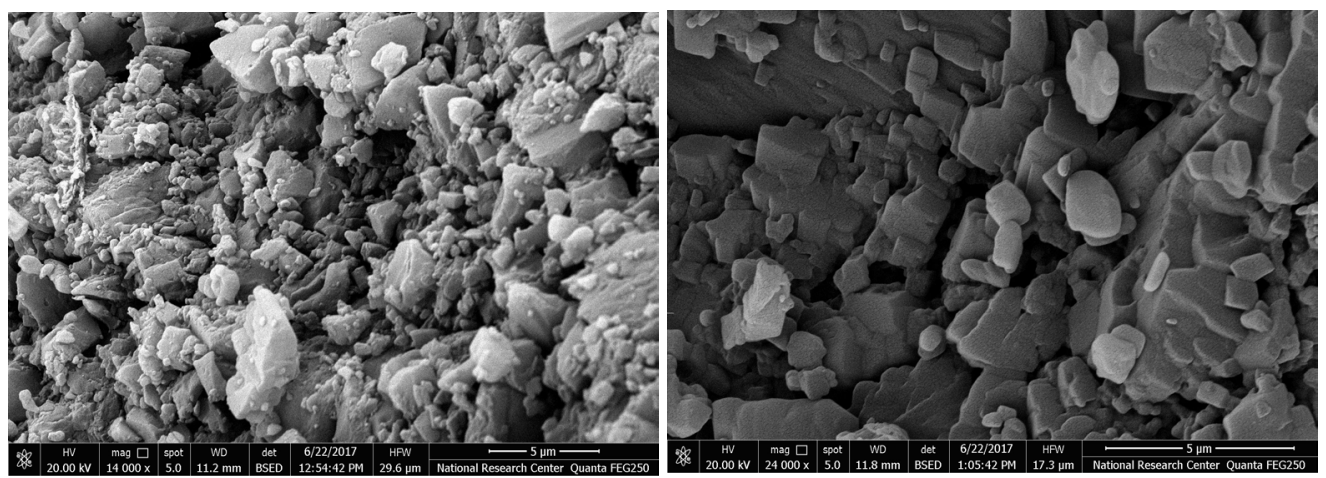

Sample 2
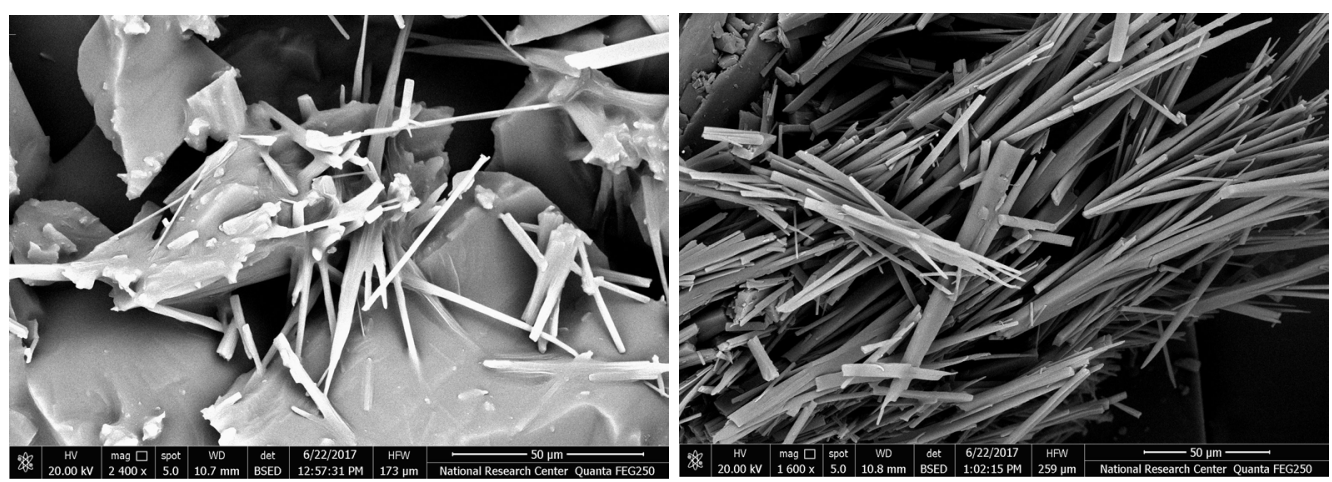

Sample 3

Figure 4. Scanning electron microscope of sample 1, 2 and 3

\subsection{The ac-electrical conductivity of studied samples $\left(\sigma_{a c}\right)$}

The ac-electrical conductivity was measured in the temperature range from 25 to $140^{\circ} \mathrm{C}$ and in a frequency range from $50 \mathrm{~Hz}$ to $5 \mathrm{MHz}$. The obtained results are plotted in Fig (5) as log versus log frequency at different temperature values. It is clear that $\sigma_{\mathrm{ac}}$ shows a steep increase with increasing frequency. At fixed temperature $40^{\circ} \mathrm{C}$ the values of electrical conductivity for sample 1,2 and 3 at frequency $100 \mathrm{~Hz}$ are $-9.3,-7.8$ and -7.7 respectively. These values increased to -5.2 , -3.4 and -2.4 respectively with increasing the frequency to $5 \mathrm{MHz}$. However with increasing temperature to $140^{\circ} \mathrm{C} \sigma_{\mathrm{ac}}$ increased to $-7.9,-5.0$ and -5.4 for sample 1,2 and 3 respectively at frequency $100 \mathrm{~Hz}$. While at same temperature $\sigma_{\mathrm{ac}}$ increases to $-5.8,-3.4$ and -2.3 for sample 1,2 and 3 respectively with increasing frequency to $5 \mathrm{MH}$. So the sample 3 is the highest conductivity due to the presence of electron withdrawal chloride ions which has negative inductive effect (-I $)^{[19]}$.

Regarding to the values of band gap calculated for the prepared samples from UV-vis spectroscopy listed previously in table (1), it is clear that it is in the range of semiconductor which mean that electrons easily move from valence band to conduction band by increasing the temperature and no longer strongly bound to specific atoms and migrate freely through the samples. This explains that the electrical conductivity increases as the temperature increases.

The dependence of ac-electrical conductivity on the frequency obeys the power low equation number (8) that related to dynamics of hopping conduction mechanism which was observed in crystalline solids ${ }^{[20]}$. 

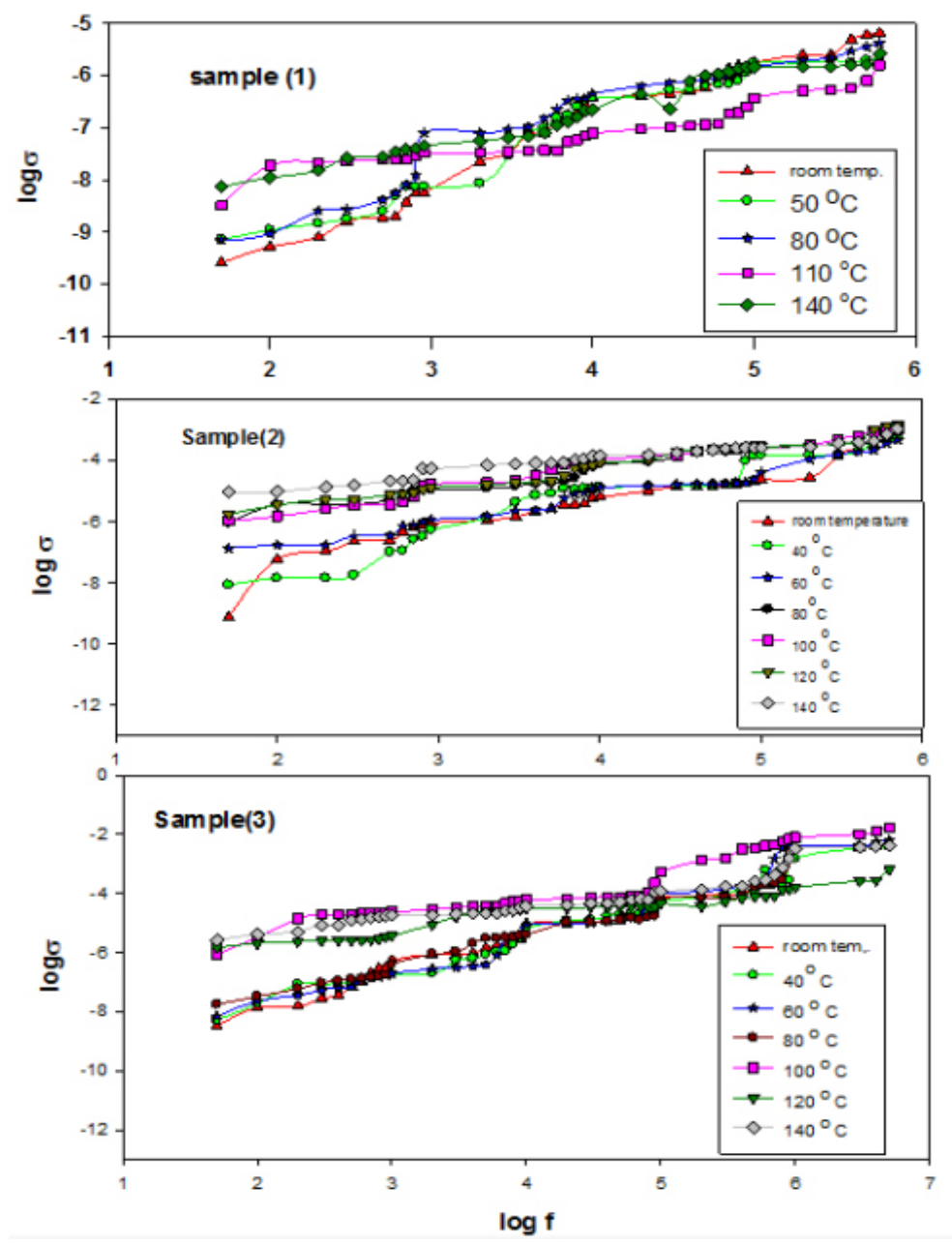

Figure 5. Ac-electrical conductivity of the prepare samples 1, 2 and 3

The values of $\mathrm{s}$ representing the degree of interaction between mobile ions and the adjacent environment ${ }^{[21]}$. $s$ was calculated from the slope of the straight line of Fig. (5). Fig. (6), represents the variation of $\mathrm{s}$ as a function of temperature. It is obvious that $\mathrm{s}$ values decrease with increasing temperature. It was previously recorded that, for ionic materials $\mathrm{s}$ values ranged from 0.6 to $1^{[22]}$. In the prepared samples we found that $\mathrm{s}$ decreases from 1.14 to 0.49 in sample 1 and from 1.16 to 0.45 of sample 2 and from 1.13 to 0.49 in sample 3 . The decrease of s values with increasing temperature can be referred to low interactions with surrounding environment that explain the higher mobility of charge carriers. A temperature dependent and its value is less than unity which suggested that the conduction goes through hopping mechanism ${ }^{[23-25]}$. However, in case of $\mathrm{s}$ values larger than unity it was reported in crystalline semiconductors ${ }^{[26,27]}$ and the motion of charge carriers goes through quantum mechanics tunneling ${ }^{[28]}$. 


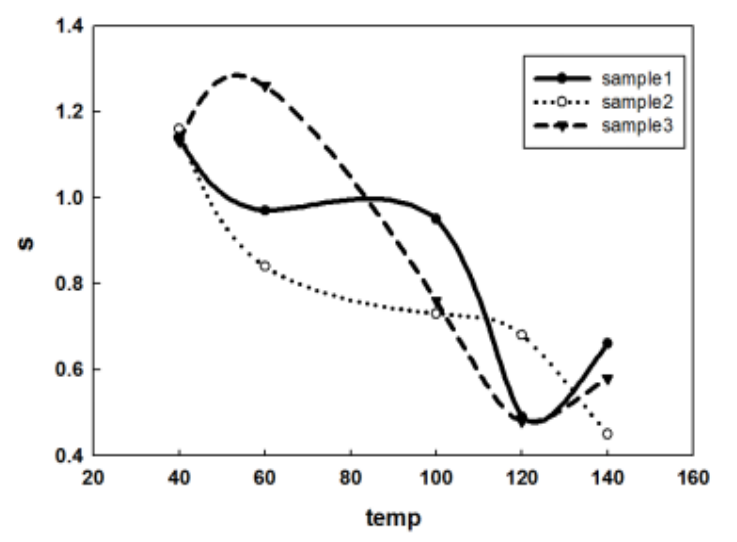

Figure 6. Variation of $S$ values with temperature of sample 1, 2 and 3

\subsection{The dc-electrical conductivity $\left(\sigma_{\mathrm{dc}}\right)$}

The dc-electrical conductivity studies help to know the mobility and activation energy of charge carriers under a dc field. The Arrhenius plot of $\log \sigma_{\mathrm{dc}}$ with $1000 / \mathrm{T}^{[29]}$ is shown in Fig. (7).

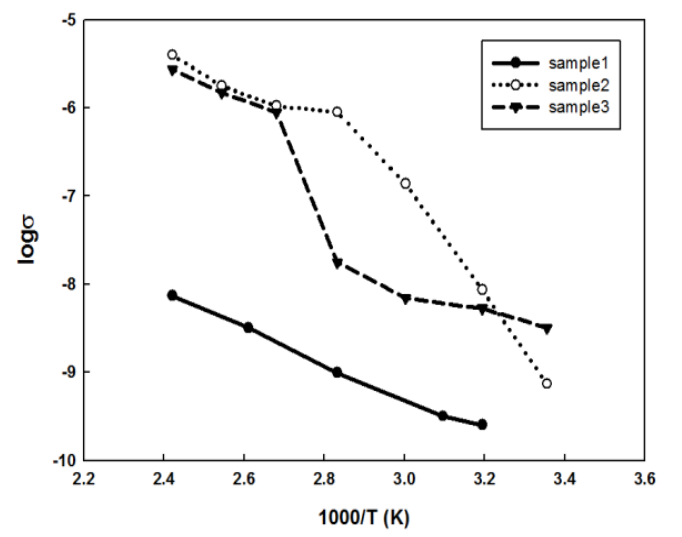

Figure 7. Variation of de-electrical conductivity with temperature

the activation energy listed in Table (2) was calculated from the slope of the plot at low and high temperature range as equations (9 and 10).

Table 2. the value of activation energy, electrical conductivity at low temperature $\sigma_{\mathrm{c}}$ and electrical conductivity $\sigma_{\mathrm{T}}$ at high temperature

\begin{tabular}{cccccc}
\hline Sample & \multicolumn{2}{c}{ dc-electrical conductivity } & \multicolumn{2}{c}{ Activation energy } \\
& $\log \sigma c(\Omega-1 \mathrm{~cm}-1)$ & $\log \sigma \mathrm{T}(\Omega-1 \mathrm{~cm}-1)$ & Low temperature & High temperature \\
\hline Sample 1 & -9.5 & -8.1 & & 0.76 \\
Sample 2 & -9.1 & -5.4 & 0.71 & 0.51 \\
Sample 3 & -8.4 & -5.6 & 0.45 & 0.42 \\
\hline
\end{tabular}

Otherwise, it is obvious that the activation energy decreases in order of sample $3<2<1$. This could be attributed to the increase of number of phenyl groups which increase the conjugation and the presence of nitrogen in sample 2 and chloride atoms in sample 3. The low value of activation energy is due to the hopping of charge carriers between localized states ${ }^{[30]}$. The appearance of two region for sample 2 and 3 which represent intrinsic and extrinsic in dc-electrical conductivity versus $1000 / \mathrm{T}$ is a sign of two types of conduction process, at low temperature less than $350 \mathrm{~K}$ is extrinsic a hopping conduction with $\Delta \mathrm{E}=0.71 \mathrm{eV}$ and $0.45 \mathrm{eV}$ for sample 2 and 3 respectively, while at higher temperature above 350 $\mathrm{K}$ is an indication of intrinsic, which is conduction thermally activated process and defects created in crystalline lattice is obvious with $\Delta \mathrm{E}=0.51 \mathrm{eV}$ and $0.42 \mathrm{eV}$ for sample 2 and 3 respectively. 


\subsection{The dielectric studies}

Dielectric study measures two fundamental electrical characteristic of materials as a function of frequency and temperature. This study measures (i) the capacitive (insulating) character, which represents its capability to store electric charge. (ii) The conductive character, which represents its ability to transfer electronic charge. From this study, the dielectric constant $\varepsilon^{\prime}$ and dielectric loss $\varepsilon^{\prime \prime}$ can be determined. In the absence of electric field, the electrons are distributed around the nuclei. When alternating electric current is applied, the electron is around in the opposite direction. Therefore, the molecules act as an electric dipole and three type of polarization were found dependent on the frequency of the electric field. Small displacement of electrons from the nucleus is electronic polarization, distortion of atomic position in a molecule is atomic polarization and the tendency of dipoles in polar molecules to align by the electric field is orientation polarization. Obviously the electronic polarization is able to follow in phase with the changing electric field compared to atomic polarization which in turn better able to follow the oscillating electric field when compared to the orientation polarization.

\subsubsection{The dielectric constant of prepared samples}

Dielectric constant is one of the essential electrical properties of solids; it measures how the electric field affects on the polarization of the materials ${ }^{[31]}$.

The variation of $\varepsilon^{\prime}$ is verified in Fig. (8). Qualitatively, $\varepsilon^{\prime}$ of the three samples has nearly the same frequency behavior where at low frequency there is a dispersion of the molecules according to the oscillation of the electron in the electric field after which at high frequency the dielectric constant is independent on frequency. Generally, the dielectric constant affected by the ionic and electronic polarization, deformation of ions and nature of the materials. The presence of delocalized electron in the phenyl ring enhanced their polarizability ${ }^{[32]}$. So the electronic structure and polarizability of oxygen in sample 1, oxygen and nitrogen in sample 2 and the addition of chloride atom in sample 3 are responsible for the variation of $\varepsilon^{\prime}$.

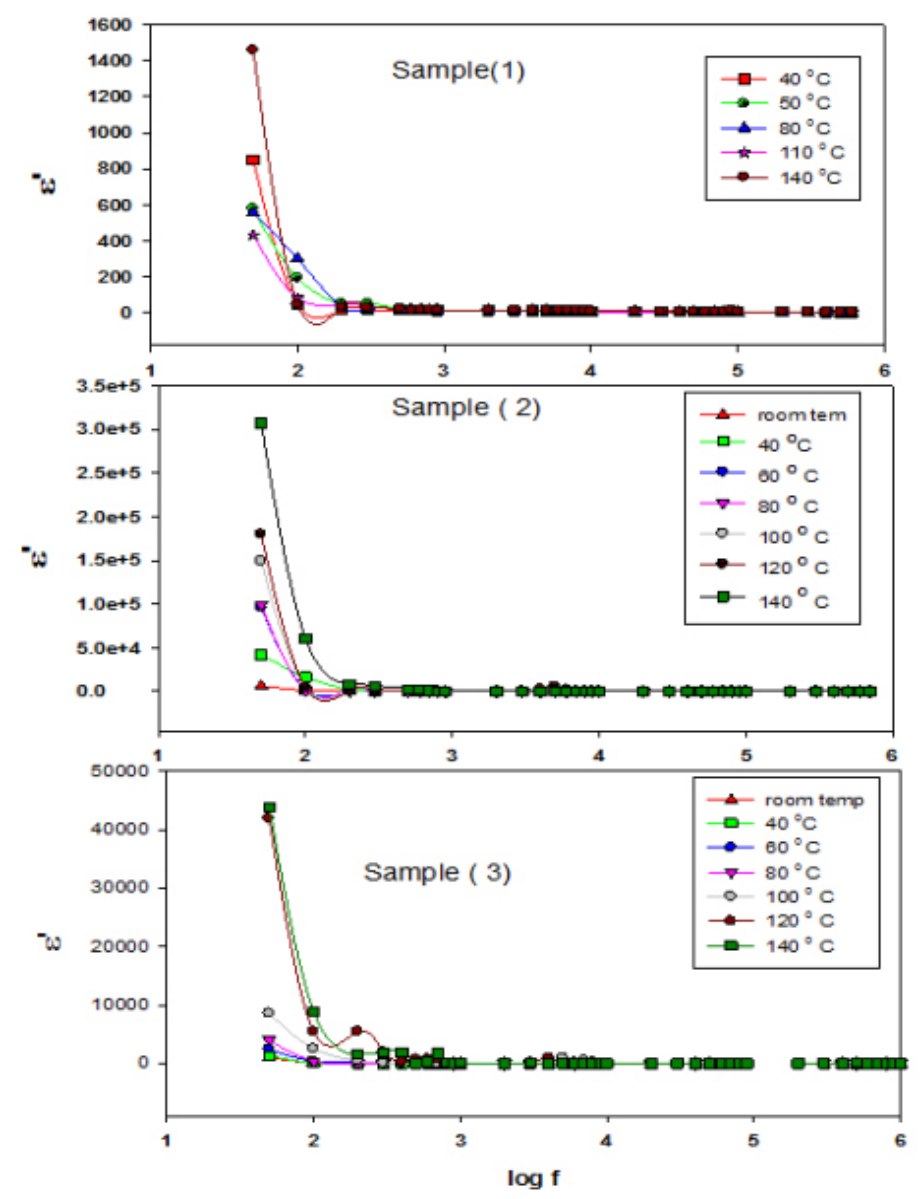

Figure 8. Variation of dielectric constant $\varepsilon^{\prime}$ with frequancy at different temperature of samples 1,2 and 3 
The values of $\varepsilon^{\prime}$ of sample $\mathbf{1}, \mathbf{2}$ and $\mathbf{3}$ at $40^{\circ} \mathrm{C}$ and $100 \mathrm{~Hz}$ are $44.9,16500$ and 138 respectively decreased to $2.9,3.1$ and 14.6 respectively at $5 \mathrm{MHz}$. However with increasing temperature to $140^{\circ} \mathrm{C}$, the dielectric constant was increased to 47.9, 60200 and 8920 respectively. At low frequency the dielectric constant has high value due to lattice vibration, dipole orientation, jump of electrons and space charge polarization ${ }^{[33]}$. With increasing temperature the electron can jump out of the sites with low free energy barriers to collect at sites with high free energy barriers in the electric field direction, this leads to polarization and therefore higher $\varepsilon^{{ }^{[34]}}$.

Fig.(9) shows the variation of dielectric constant $\varepsilon^{\prime}$ as a function of temperature at radio frequency $1 \mathrm{KHz}$ and microwave frequency $1 \mathrm{M} \mathrm{Hz}$. At $1 \mathrm{M} \mathrm{Hz}$ the variation is the same in all samples. While at low frequency the dependence is weak over the temperature range of sample 1 , up to $40^{\circ} \mathrm{C}$ for sample 2 and up to $80^{\circ} \mathrm{C}$ for sample 3 .
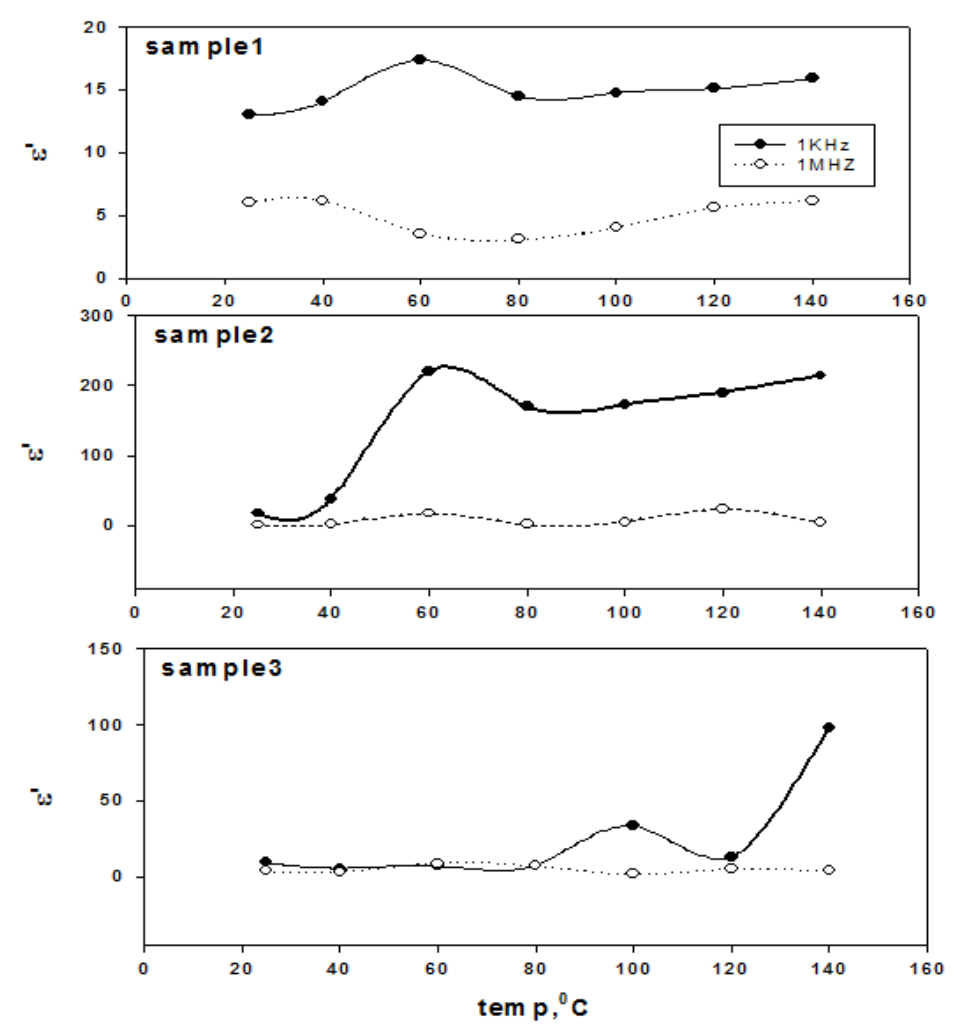

Figure 9. variation of dielectric constant of sample 1,2 and 3 with temperature at frequency $1 \mathrm{~K} \mathrm{~Hz}$ and I M Hz

It is worth to note that for the increase of $\varepsilon^{\prime}$ of sample 2 above $40^{\circ} \mathrm{C}$ and then followed by a decrease at $60^{\circ} \mathrm{C}$ then increase again with increasing temperature above $80^{\circ} \mathrm{C}$ as shown in Fig. (9) May be due to nomadic polarization, which arises from the extreme increase in the number of free carriers with increasing temperature and contribution of more number of dipoles and inter ionic forces of the crystal which become weak ${ }^{[35]}$.

Certain structures display a higher polarization than the others. The presence of highly polarized aromatic rings increase $\varepsilon^{\prime}$. The $\pi$ bond in the aromatic rings is loosely attached compared to the sigma bond therefore it is easily polarized. For large size_atoms like bromine and iodine, the electron cloud is so large and further independent of the influence of electrostatic attraction of the positive nucleus. It is expected to display a high polarizibility. This is as oppose chloride which has small atomic radius $\left(1.6 \mathrm{~A}^{\circ}\right)$ and intense negative charge. It is able to hold the electron cloud much tightly resulted in a low polarizability. This will induce a lower dielectric constant as recorded for sample 3 which has less dielectric constant value than sample 2. However, at high frequency, the charge carriers cannot relax as rapidly as the time variation of the field, so the charge swinging lag behind this field, leads to decrease of $\varepsilon^{\prime}$ which enables these compounds to be suitable for electro-optic applications ${ }^{[36]}$.

3.6.2 The dielectric loss $\left(\varepsilon^{\prime \prime}\right)$

Dielectric loss $\varepsilon^{\prime \prime}$ similar to dielectric constant $\varepsilon^{\prime}$ depends on temperature and frequency of applied electric field. Dielectric loss $\varepsilon$ " is displayed in Fig.(10). At low frequency and all temperature, a strong dependence is observed, while in 
sample 2 at higher frequency $\varepsilon^{\prime \prime}$ approaches to a constant and low values. The electronic structure and charge accumulation will perform an important role in $\varepsilon^{\prime \prime}$ in this organic compounds. At high frequency region, an absorption maximum peak in the dielectric loss spectra is high due to the accumulation of ionic charges ${ }^{[37]}$.
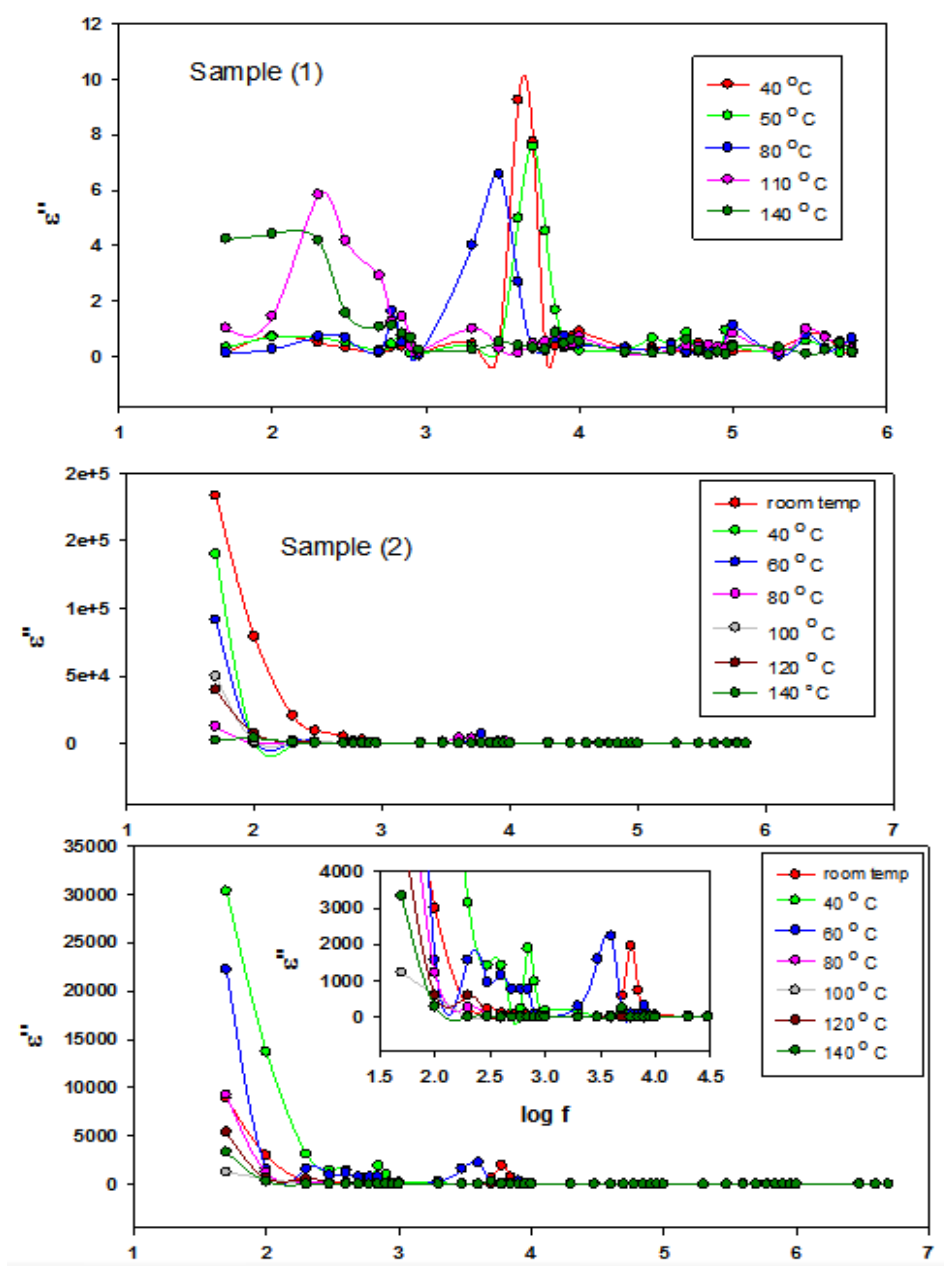

Figure 10. Variation of dielectric loss with frequency at different temperat of samples1, and 3

The relaxation time $(\tau)$ was calculated using equation $(5)^{[38]}$. For sample 1 and 3 the value of $\tau$ depends on frequency and temperature. The enthalpy and the entropy of the relaxation process are calculated by using usual rate equation (6). The enthalpy $\Delta \mathrm{H}$ is determined from the slopes and entropy $\Delta \mathrm{S}$ from the intercept of the fitted lines of $\ln (\mathrm{kT} \tau / \mathrm{h})$ and the reciprocal of the temperature. The values of relaxation time, enthalpy $\Delta \mathrm{H}$ and entropy $\Delta \mathrm{S}$ listed in Table (3)

Table 3. The apparent relaxation times $\tau$, the activation enthalpies $\Delta H$ and the entropy changes $\Delta S$ for sample 1 and 3

\begin{tabular}{ccccccccc}
\hline & \multicolumn{4}{c}{$\log \tau$} & \multicolumn{3}{c}{$\begin{array}{c}\mathrm{AH} \\
\mathrm{KJ} / \mathrm{mole}\end{array}$} & $\begin{array}{c}\mathrm{AS} \\
\mathrm{J} / \mathrm{deg} / \mathrm{mole}\end{array}$ \\
\hline Sample 1 & 0.044 & 50 & 60 & 80 & 110 & 140 & \\
Sample 3 & 0.049 & 0.056 & $-0 .------$ & 0.046 & 0.068 & 0.079 & $2.4 \times 10^{3}$ & -59.7 \\
\hline
\end{tabular}

The dielectric relaxation time of sample 1 and 3 was calculated from the maximum absorption bands. The activation enthalpies $\Delta \mathrm{H}$ for sample $\mathbf{1}$ is $2.4 \times 10^{3} \mathrm{KJ} / \mathrm{mole}$ and $4.4 \times 10^{3} \mathrm{KJ} / \mathrm{mole}$ for sample 3 as listed in table (3). The values of the entropy changes of dielectric relaxation $\Delta \mathrm{S}$ were -59 and -77.15 . The values of entropy are negative indicating that the relaxation process is the rate determining factor.

\section{Summary and conclusions}

In this work, the cyclopentenone derivatives were prepared. Structural analysis was carried out for all the studied 
samples using XRD patterns and SEM explains that all the studied compounds have a definite crystalline structure. The band gap energies were calculated from UV-Vis. spectra and its values are 2.86, 2.6 and 2.67 for sample 1, 2 and 3 respectively.

The capacitance data of cyclopentenone derivatives were employed to study their dielectric properties. Electrical properties as dc- and ac-electrical conductivity are affected greatly by temperature and frequency.

The increase of conductivity with frequency may be due to decrease in space charge polarization. The frequency and temperature dependent $\sigma_{\mathrm{ac}}$ obey the power law. The exponent $\mathrm{s}$ is less than unity and decreases with increasing temperature. Analysis of $\sigma_{\mathrm{ac}}$ data signifies that the correlated barrier hopping $(\mathrm{CBH})$ model is the most suitable mechanism for $\sigma_{\mathrm{ac}}$ behavior. And when s is more than unity the tunneling is suitable mechanism. The values of activation energy of samples 1,2 and 3 were calculated by Arrhenius equation and have the values of $0.76,0.71$ and $0.45 \mathrm{eV}$ respectively and at high temperature is 0.51 and $0.42 \mathrm{eV}$ for sample 2 and 3 respectively.

Qualitatively, the behavior of the dielectric constant of three samples as a function of temperature and frequency were similar, but with difference in their values. This variation in the values might be due to its structure and accumulation of charges in the sample. The dielectric relaxation time, activation enthalpy and entropy were calculated for samples $\mathbf{1}$ and $\mathbf{3}$.

\section{Acknowledgement}

The authors thank the National Research Centre for financial support (project number 11090305).

\section{References}

[1] D. W. Samuel, G. A. Turnbull. Organic semiconductor Lasers. Chem Rev. 2007; 107(4): 1272-1295.

[2] S. Chenais, S. Forget. Recent Advances in Solid State Organic Lasers. Polym. Int. 2012; 61(3): 390-406.

[3] J.C. Anderson. Dielectrics. Chapman and Hall, London: 1964.

[4] L. Akella, R. Vince. Enantioselective Synthesis of 2-[5-(9H-purine-9-yl)-2-cyclopenten-1-yl]-ethanol analogus as potential antiviral agents. Tetrahedron. 1996; 52(25): 8407-8412.

[5] S. E. Gibson, S. E. Lewis, N. Mainolfi. Transition Metal - Mediated Routes to cyclopentenones. J. Organomet. Chem. 2004; 689(24): 3873.

[6] M. Nardi, P. Costanzo, A. De.Nino, M. L. DiGioia, F. Olivito, G. Sindona. A. Procopio, water Excellent Solvent for the Synthesis of bifuntionalized cyclopentenones from furfural. Green Chemistry. 2017; 1-9.

[7] S. Fustero, M. Sanchez-Rosell, P. Barrio, A. Simn-Fuentes. A Furitful Decade for the synthesis of Pyrazoles. Chem Rev. 2011; 111(11): 6984-7034.

[8] A. Balbi, M. Anzaldi, M. Mazzei, M. Miele, M. Bertolotto, L. Ottonellob, F. Dallegrib. Synthesis and biological evaluation of novel heterocyclic ionone- like derivatives as anti- inflammatory agent. Bioorg Med Chem. 2006; 14(15): 5152-5160.

[9] J. Zaumseil, H. Sirringhaus. Electron and Ambipolar Transport in Organic Field- Effect Transistors. Chem Rev. 2007; 107: 1296-1323.

[10] K. Walzer, B. Maennig, M. Pfeiffer, K. Leo. Highly Efficient Organic Devices Based on Electrically Doped Transport Layers. Chem. Rev. 2007; 107: 1233-1271.

[11] C. J. Brabec, N. S. Sariciftci, J. C. Hummelen. Plastic Solar Cells. Adv. Funct. Mater. 2001; 11: 15-26.

[12] X. C. Wang, M. Maeda, A. Thomas, K. Takanabe, G. Xin, J. M. Carlsson, K. Domen, M. Antonietti. A Metal-Free Polymeric Photocatalyst for Hydrogen Production from Water under Visible Light. Nat Mater. 2009; 8: 76-80.

[13] K. Hayashi, S. Matsuishi, T. Kamiya, M. Hirano, H. Hosono. Light-Induced Conversion of an Insulating Refractory Oxide into a Persistent Electronic Conductor. Nature. 2002; 419: 462-465.

[14] V. Mikhura, A. A. Formanovskii, A. S. Shashkov. Structure of 2(5)- alkyl-4-hydroxy-3,4-diphenylcyclopent-2-en-1ones, cyclocondensation products of benzil with aliphatic ketones. Russ J. Org Chem. 2010; 46 (8): 1116-1120.

[15] F. A. El-Samahy. Synthesis of New Pyrazoles via Cycloaddition Reactions of Nitrilimines with 2-Cyclopentenone Derivatives. J. Heterocyclic Chem, 2015; 52(2): 597-602.

[16] F. A. El-Samahy· A. M. Abd Elkarim, M. S. El-Sedik, A. H. Salama · F. H. Osman. Correlation between Semiconductor Characteristics and Molecular Structure of New Types of Substituted 1, 3, 5-Triazine Derivatives. Silicon. 2018; 10: 659-665.

[17] B. C. Smith. Quantitative Spectroscopy: Theory and Practice. Amsterdam: Elsevier; 2002.

[18] M. Hoffman, S. Martin, W. Choi, D. Bahnemann Environmental applications of semiconductor photo catalysis Chemical Review. 1995; 69.

[19] A. Patterson. The Scherrer Formula for X-Ray Particle Size Determination. Phys Rev. 1939; 56(10): 978-982. 
[20] W. M. Darwish, M. A. Abd El Ghaffar, G. M. Turky. Synthesis and Electric Modulus Formalism of Novel MetalPhthalocyanine Bridged Polymers J. Inorg. Organomet .polym. 2014; 24: 858-864.

[21] S. Suresh, K. Anand. Studies on optical, dielectric and electrical conductivity properties of zinc succinate NLO single crystal. Advances in Applied Science Research. 2012; 3(2): 815-820.

[22] R.H. Chen, L.-F. Chen, C.-T. Chia. Impedance spectroscopic studies on congruent LiNbO3 single crystal. J. Phys. Condens. Matter. 2007; 19: 086225.

[23] W.K. Lee, J.F. Liu, A.S. Nowick. Limiting behavior of ac conductivity in ionically conducting crystals and glasses, A new universality. Phys. Rev. Lett. 1994; 67: 1559.

[24] M.M.D. Lima, R. Borsali. Rodlike cellulose microcrystals: structure, properties, and applications, Macromol. Rapid Commun. 2004; 25: 771-787.

[25] J.C. Dyre, T.B. Shrøder. Universality of ac conduction in disordered solids. Rev. Mod. Phys. 2000; 72: 873-89.

[26] G.C. Psarras. Hopping conductivity in polymer matrix-metal particles composites. Compos. Part A 2006;37: 15451553.

[27] S.R. Elliott. A.c. conduction in amorphous chalcogenide and pnictide semiconductors. Adv. Phys. 1987;36 (2): 135217. S.R. Elliott. Frequency-dependent conductivity in ionically and electronically conducting amorphous solids Solid State Ion. 1994; 70-71, 27-40.

[28] H.M. El-Mallah, N.A. Hegab. Studies on a.c properties of Ca1-xSrxTiO3 Perovskites. J. Mater. Sci. 2007; 42(1): 332336.

[29] K.S. Gilroy, W.A. Phillips. An Asymmetric double - well potential model for structural relaxation processes in amorphous materials. Philos Mag. 1981; B 43(5): 735-746.

[30] S. P. Mondal, R. Aluguri, S. K. Ray. Dielectric and transport properties of carbon nanotube- CdS nanostructures embedded in polyvinyl alcohol matrix. J. of Appl. Phys. 2009; 105: 114317.

[31] H. M. Zeyada, M. M. El-Nahass. Electrical properties and dielectric relaxation of thermally evaporated zinc phthalocyanine thin films. Appl. Surf. Sci. 2008; 254(6): 1852.

[32] C.P. Smyth, Dielectric behaviour and structure. Megraw Hill, New York: 1965.

[33] F. Tsunesada, T. Iwai, T. Watanabe, H. Adachi, M. Yoshimura, Y. Mori, T. Sasaki. High-quality crystal growth of organic nonlinear optical crystal DAST. J. Cryst. Growth. 2002; 237-239: 2104-2106.

[34] S.K. Arora, V. Patel, B. Amin, A. Kothari. Dielectric behaviour of strontium tartarate crystals. Bullettin of Material Science, 2004; 27: 141-147.

[35] R. S. Kumar, K. Hariharan. AC conductivity and electrical relaxation studies on 10CuI-60AgI-30V2O5 glasses. Mat. Chem. Phys. 1999; 60(1): 28.

[36] H. S. Nalwa, P. Vazudevan. Dielectric properties of cobalt phthalocyanine. J. Mat. Sci. Lett. 1983; $2(1): 22$.

[37] P. Bergo, W. M. Pontuschka, J. M. Prison, C. C. Motta, J. R. Martinelli. Dielectric properties of barium phosphate glasses doped with transition metal oxides. J Non-Cryst. Solids. 2004; 348: 84-89.

[38] A. M. Stephan, T. Premkumar, N. G. Renganathan, S. Pitchumani, R. Thirunakaran, N. Muniyandi. Ionic conductivity and FT-IR studies on plasticized PVC/PMMA blend polymer electrolytes. Journal of Power Sources. 2000; 89: 80-87.

[39] S. Glasstone, K. J. Laidle, H. Eyring. The Theory of Rate Processes. McGraw-Hill, New York:1941. 